\title{
Workspace Analysis of A 2-DOF Planar Parallel Mechanism
}

\author{
Y.Y. Chen, X.Z. Han, F. Gao, Z.H. Wei, Y. Zhang \\ School of Mechanical and Precision Instrument Engineering \\ Xi'an University of Technology \\ Xi'an, China
}

\begin{abstract}
A 2- Degree Of Freedom (DOF) planar parallel mechanism is introduced and the inverse solution of the kinematics and Jacobin matrix and workspace analysis are carries out. The mathematical conditions of which the singular configuration of the manipulator occurred based on Jacobin matrix and the relationship between the singular configuration and the bars are obtained. The impacts of design parameters on workspace are analysed and the workspace is discussed in detail using the graphical method, and the sizes of area on different design parameters are derived. The results are useful to the manufacturing and control of 2-DOF parallel prototype.
\end{abstract}

Keywords- parallel mechanism; kinematic analysis; singularity; workspace analysis

\section{INTRODUCTION}

Parallel mechanisms have advantages in dynamic performance, stiffness, accuracy, payload capability and have aroused the great interest of a wide variety of researchers [1]. As research continues, the weaknesses and shortages of 6DOF Parallel mechanism are exposing, such as strong coupling in position and posture space, weak posture performance of the moving platform, no explicit kinematic forward solution, complicated design and control [2].

Compared with the general 6-DOF parallel mechanism, lower-mobility parallel mechanisms [1,3] whose degrees of freedom are less than six have advantages of simple structure, low cost in design, manufacturing and control. Because of its broad application prospects, lower-mobility parallel mechanisms are becoming the focus and emphasis of the research[4]. The 2-DOF parallel mechanism is a very important member of lower-mobility parallel mechanisms family and the planar parallel mechanism of the 2-DOF parallel mechanism can realize accurate positioning and arbitrary trajectory tracking in a plane. Many scholars have been engaged in it, and have reaped rich fruits [5-8]. In this article, a planar 2-DOF parallel mechanism is proposed and the kinematics, singularity and workspace are studied systematically.

\section{KINEMATIC ANALYSIS}

\section{A. Inverse Kinematics Analysis}

The proposed 2-DOF parallel mechanism illustrated in fig. 1 is composed of a fixed base platform, a moving platform, two active sliders and two kinematic chains. One of the two kinematic chains consists of a parallelogram. Sliders $A_{1}$ and
$\mathrm{A}_{2}$ drive the two kinematic chains when they slide along the guide ways; and the sliders are driven by the servomotors via leads screw. Thus the moving platform possesses a 2-DOF translational moving capability in a plane.

As showed in fig. 1 , the base coordinate system $O-X Z$ is attached to the base and the moving coordinate system $O_{m}-X_{m} Z_{m}$ is fixed at the center of the compound rotating joints. The distance between the two parallel sliders e is $a$.

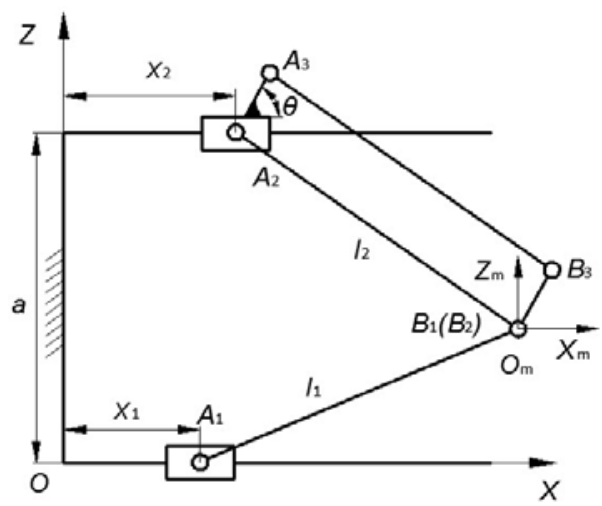

FIGURE I. SCHEMATIC DIAGRAM OF THE PLANAR 2-DOF PARALLEL MECHANISM

According to the geometric relationship, the constraint equation associated with the links can be written as:

$$
\left|A_{i} B_{i}\right|=l_{i}(i=1,2)
$$

In coordinate system $O-X Z$, the point coordinate of $A_{i}(i=1,2)$ and $B_{i}(i=1,2)$ are $\left(X_{i}, 0\right)$ and $(X, Z)$.

From eqn(1), the following equations can be obtained.

$$
\left\{\begin{array}{l}
\left(X-x_{1}\right)^{2}+Z^{2}=l_{1}^{2} \\
\left(X-x_{2}\right)^{2}+(Z-a)^{2}=l_{2}^{2}
\end{array}\right.
$$

The inverse kinematic solutions of the parallel mechanism can be written as

$$
\left\{\begin{array}{l}
x_{1}=X \pm \sqrt{l_{1}^{2}-Z^{2}} \\
x_{2}=X \pm \sqrt{l_{2}^{2}-(Z-a)^{2}}
\end{array}\right.
$$


From the form of the inverse position solution , the parallel mechanism has four working modes which showed in fig. $2 \mathrm{a}(+,+), 2 \mathrm{~b}(+,-), 2 \mathrm{c}(-,-), 2 \mathrm{~d}(-,+)$.
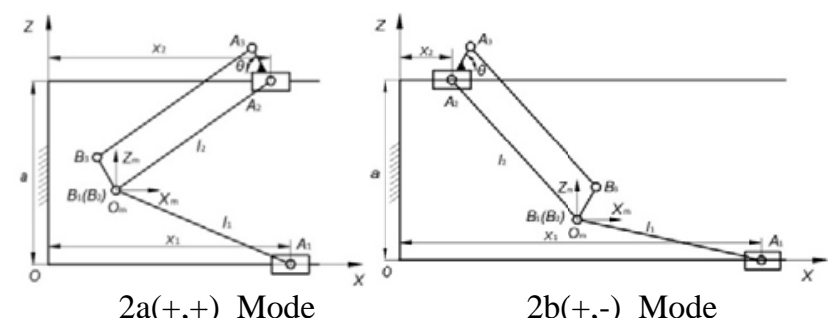

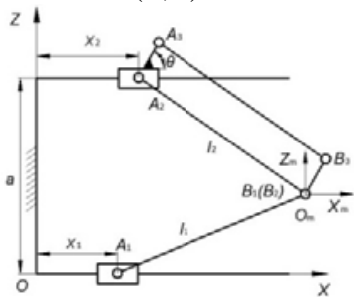

2c(-,-) Mode

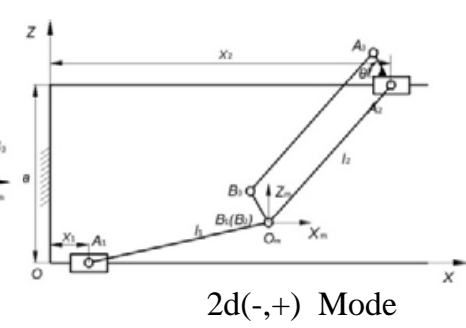

FIGURE II. THE WORKING MODE.

\section{JACOBIAN MATRIX AND SINGULARITY ANALYSIS}

\section{A. Jacobian Matrix}

Taking the time derivative of eqn(2) lead to

$$
J_{q} \dot{q}=J_{\mathrm{X}} \dot{X}
$$

Where

$$
\begin{gathered}
\boldsymbol{J}_{\boldsymbol{q}}=\left[\begin{array}{cc}
X-x_{1} & 0 \\
0 & X-x_{2}
\end{array}\right], \dot{\boldsymbol{q}}=\left[\begin{array}{c}
\dot{X}_{1}, \dot{X}_{2}
\end{array}\right]^{T}, \boldsymbol{J}_{X}=\left[\begin{array}{cc}
X-x_{1} & Z \\
X-x_{2} & Z-a
\end{array}\right], \\
\dot{\boldsymbol{X}}=[\dot{X}, \dot{Z}]^{T} .
\end{gathered}
$$

If $\left|\boldsymbol{J}_{\boldsymbol{X}}\right| \neq 0$, the Jacobian matrix of the parallel mechanism can be obtained.

$$
\boldsymbol{J}(\boldsymbol{q})=\boldsymbol{J}_{X}^{-1} \boldsymbol{J}_{\boldsymbol{q}}=\left[\begin{array}{cc}
\frac{\left(X-x_{1}\right)(a-Z)}{Q} & \frac{Z\left(X-x_{2}\right)}{Q} \\
\frac{\left(X-x_{2}\right)\left(X-x_{1}\right)}{Q} & -\frac{\left(X-x_{2}\right)\left(X-x_{1}\right)}{Q}
\end{array}\right]
$$

Where $Q=a X+Z x_{1}-a x_{1}-z x_{2}$

\section{B. The Singularity Analysis}

Because singularities lead to a loss of the controllability and degradation of the natural stiffness of manipulators, they must be avoided in the task workspace. In the parallel mechanism, singularities occur whenever $\boldsymbol{J}_{\boldsymbol{q}}, \boldsymbol{J}_{\boldsymbol{X}}$ or both, become singular.

The boundary singularity occurs when $\boldsymbol{J}_{\boldsymbol{q}}$ becomes singular but $\boldsymbol{J}_{\boldsymbol{X}}$ remains invertible. This corresponds to the configuration whenever one of the legs $l_{1}$ or $l_{2}$ is perpendicular to the $\mathrm{X}$-axis. The configurations of this kind of singularity are shown by the dotted line in fig.3.
The singularity of internal workspace arises when $\boldsymbol{J}_{\boldsymbol{X}}$ becomes singular but $\boldsymbol{J}_{\boldsymbol{q}}$ remains invertible. This corresponds to the configuration whenever the legs $l_{1}$ and $l_{2}$ are collinear but not perpendicular to the $\mathrm{X}$-axis. The configurations of this kind of singularity are shown by the solid line in fig.3.

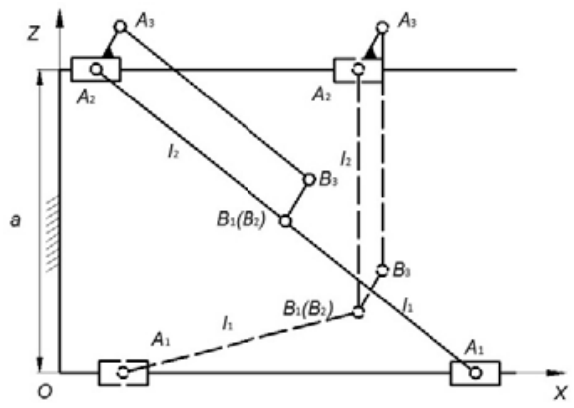

FIGURE III. THE BOUNDARY SINGULARITY.

\section{WORKSPACE ANALYSIS}

The workspace which determines the reachable region of the mechanism is one of the most important kinematics properties of parallel mechanism. For the parallel mechanisms of different structure, acquiring the changes and distributions of workspace is the indispensable condition for trajectory planning, and prerequisite and foundation for optimal design based on the performance of the workspace. The approaches to determine workspace of a parallel mechanism are analytical , numerical and graphing method [9]. From an engineering perspective, analytical method is very complicated and the intuition is not strong. Numerical method is used for obtaining the workspace based on forward and inverse position solution, and the forward position solution is the common methods to determine the workspace of traditional serial mechanism. But due to the forward position solution of the parallel mechanism is generally complex, the inverse solution is the common methods to obtain the workspace, not the forward solution [10]. For the 2-DOF parallel mechanism studied in this paper, the mapping method which can directly reflect the distribution of the workspace is a simple and practicable method and is adopted in this paper.

Given the stroke $\delta$ of sliders $A_{1}$ and $A_{2}$. The theoretical workspace which is shown in fig. 4 of the parallel mechanism is obtained when the sides slide along the guide ways .

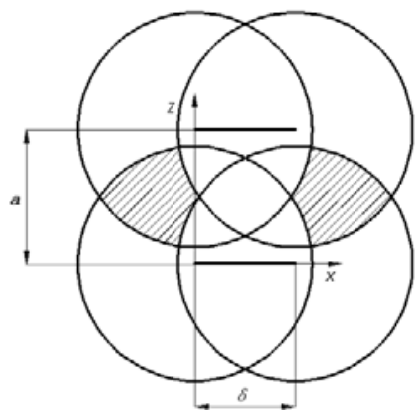

FIGURE IV. THEORETICAL WORKSPACE DIAGRAM. 


\section{A. Effective Workspace}

Here Select the working mode which is shown in fig 2c (-, -) to study. The area and shape of the effective workspace vary with the changes of the parameters $\delta, a$ and $l\left(l_{1}=l_{2}\right)$.

1) $0<\delta<l$

a) $0<a<l$.In this case the shapes of the effective workspace are shown in fig.5. And when the parameters $\delta$, $a$ and $l$ suit for the relationships as follows :

(1) $a^{2}+\delta^{2}-2 \delta l>0$, the shapes of the effective workspace are shown in fig.5(a-1) and the value of the area

$$
S=2 \int_{a / 2}^{b}\left(\sqrt{l^{2}-z^{2}}+\delta-\sqrt{l^{2}-(z-a)^{2}}\right) d z
$$

Where

$b=\frac{1}{2} \frac{a^{3}+a \delta^{2}+\sqrt{4 a^{2} \delta^{2} l^{2}+4 \delta^{4} l^{2}-2 \delta^{4} a^{2}-a^{4} \delta^{2}-\delta^{6}}}{a^{2}+\delta^{2}}$

the parameter of $b$ has the same meaning in the equation below.

(2) $a^{2}+\delta^{2}-2 \delta l=0$, the shapes of the effective workspace are shown in fig.5(a-2) and the value of the area

$$
S=2 \int_{a / 2}^{a}\left(\sqrt{l^{2}-z^{2}}+\delta-\sqrt{l^{2}-(z-a)^{2}}\right) d z
$$

(3) $a^{2}+\delta^{2}-2 \delta l<0$, the shapes of the effective workspace are shown in fig.5(a-3) and the value of the area

$$
S=2 \int_{a / 2}^{a}\left(\sqrt{l^{2}-z^{2}}+\delta-\sqrt{l^{2}-(z-a)^{2}}\right) d z
$$

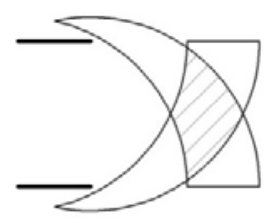

(a-1)

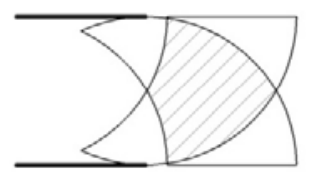

(b)

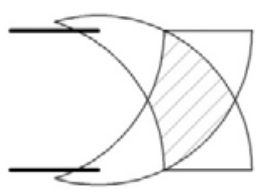

(a-2)

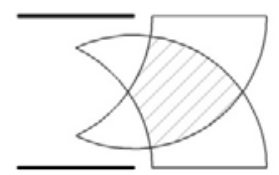

(c)
FIGURE V. THE SHAPE OF EFFECTIVE WORKSPACE $(0<\delta<l$ ).

b) $a=l$. In this case the shapes of the effective workspace are shown in fig .5(b) and the value of the area

$$
S=2 \int_{a / 2}^{b}\left(\sqrt{l^{2}-z^{2}}+\delta-\sqrt{l^{2}-(z-a)^{2}}\right) d z
$$

c) $a>l$. In this case the shapes of the effective workspace are shown in fig.5(c) and the value of the area

$$
S=2 \int_{a / 2}^{b}\left(\sqrt{l^{2}-z^{2}}+\delta-\sqrt{l^{2}-(z-a)^{2}}\right) d z
$$

2) $\delta=l$. In this case the shapes of the effective workspace are shown in fig.6. And when parameters $a$ and $l$ suit for the relationships as follows:

a) $0<a<l$. In this case the shapes of the effective workspace are shown in fig.6(a)and the value of the area

$$
S=2 \int_{a / 2}^{a}\left(\sqrt{l^{2}-z^{2}}+\delta-\sqrt{l^{2}-(z-a)^{2}}\right) d z
$$

b) $a=l$. In this case the shapes of the effective workspace are shown in fig.6(b) and the value of the area

$$
S=2 \int_{a / 2}^{l}\left(\sqrt{l^{2}-z^{2}}+\delta-\sqrt{l^{2}-(z-a)^{2}}\right) d z
$$

c) $a>l$. In this case the shapes of the effective workspace are shown in fig.6(c) and the value of the area

$$
S=2 \int_{a / 2}^{l}\left(\sqrt{l^{2}-z^{2}}+\delta-\sqrt{l^{2}-(z-a)^{2}}\right) d z
$$

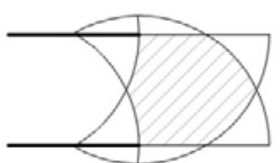

(a)

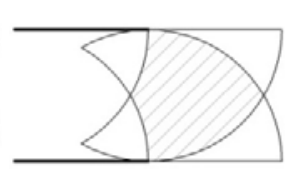

(b)

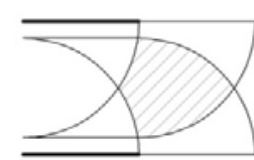

(c)
FIGURE VI. THE SHAPE OF EFFECTIVE WORKSPACE ( $\delta=l$ ).

3) $\delta>l$. In this case the shapes of the effective workspace are shown in fig.7. And when parameters $a$ and $l$ suit for the relationships as follows:

a) $0<a<l$. In this case the shapes of the effective workspace are shown in fig .7(a) and the value of the area

$$
S=2 \int_{a / 2}^{a}\left(\sqrt{l^{2}-z^{2}}+\delta-\sqrt{l^{2}-(z-a)^{2}}\right) d z
$$

b) $a=l$. In this case the shapes of the effective workspace are shown in fig .7(b) and the value of the area

$$
S=2 \int_{a / 2}^{l}\left(\sqrt{l^{2}-z^{2}}+\delta-\sqrt{l^{2}-(z-a)^{2}}\right) d z
$$

c) $a>l$. In this case the shapes of the effective workspace are shown in fig.7(c) and the value of the area

$$
S=2 \int_{a / 2}^{l}\left(\sqrt{l^{2}-z^{2}}+\delta-\sqrt{l^{2}-(z-a)^{2}}\right) d z
$$

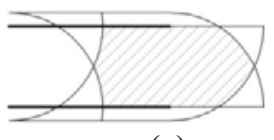

(a)

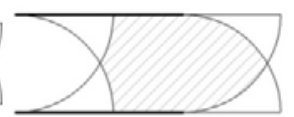

(b)

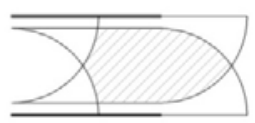

(c)
FIGURE VII. THE SHAPE OF EFFECTIVE WORKSPACE $(\delta>l)$.

\section{CONCLUSIONS}

The paper presented a 2-DOF planar parallel mechanism and its inverse kinematic and the Jacobian analysis are obtained. The effects of the changes of various parameters on 
its workspace are demonstrated in detail and the conclusions are as follows:

1) With other conditions unchanged the larger the travel of the guide way is, the larger the workspace is.

2) The structure of variable linkages can improve the applicability of the parallel mechanism.

\section{ACKNOWLEDGMENTS}

This work was supported by Science Research Program of Shanxi Province (Grant No. 2013JQ7009), Department of Education research project of Shanxi Provincial (Grant No. 14JK1527).

\section{REFERENCES}

[1] MERLET J P. Parallel robots [M].Dordrecht, The Netherlands: Kluwer Academic Publishers,pp.1-20,2000.

[2] X.L. Yu, Y.W. Liu \& L.H. Chu, Analysis of degree of freedom and motion characteristics for a kind of parallel mechanism with few degree of freedom. Journal of Machine Design,23(2), pp .10 12,2006.

[3] Z. Huang, Y.S. Zhao \& T.S. Zhao, Advance Spatial Mechanism. Beijing : Higher Education Press,pp.89 210. 2006

[4] H.Y Ruan \& Y.H. Wang, The Research Situation and Prospection of Key Technology and Theory for Lower-freedom Parallel Structure. Machine Tool \&Hydraulics, 39(13),pp.133 136,2011.

[5] F. Gao, W. Li, X. Zhao, Z Jin \& H Zhao, New kinematic structures for 2,3-,4-, and 5-DOF parallel manipulator designs. Mechanism and machine theory, 37(11),pp.1395-1411,2002

[6] J. Wu, T.M. Li, X.J. Liu \& L.P. Wang. Optimal Kinematic Design of a 2DOF planar parallel manipulator. Tsinghua University(Sci. \& Technol.),12(3), pp.269-275,2007.

[7] J. Wu, J.S. Wang, T.M. Li, L.P. Wang \& L.W. Guan, Dynamic dexterity of a planar 2-DOF parallel manipulator in a hybrid machine tool. Robotica, Cambridge Univ Press,26(1),pp.93-98,2008 .

[8] X.J. Liu, J.S. Wang \& G Pritschow, On the optimal kinematic design of the PRRRP 2-DoF parallel mechanism. Mechanism and Machine Theory, 41(9) ,pp.1111-1130,2006.

[9] X.R. Zhu, H.P. Shen \& C.F. Liu, Workspace and Singularity Analysis of a 2-DOF Parallel Manipulator. Machine Design and Research, 26(5), pp.31 35, 2010.

[10] X.X. Gao, Y. Mei\& R.Q. Li. Analysis of Workspace of the 3-PPR Planar Parallel Manipulator. Journal of Mechanical Transmission,38(2),pp. 90 92,2014. 